\title{
Similar Pattern Discrimination by Filter Mask Learning with Probabilistic Descent
}

\author{
Yoshiaki Kurosawa \\ Toshiba Solutions Co., Advanced Technology Development, Platform Solutions Div. \\ 1-15 Musashidai, 1, Fuchu-shi, Tokyo 183-8532, Japan
}

\begin{abstract}
The purpose of this research was to examine the learning system for a feature extraction unit in OCR. Average Risk Criterion and Probabilistic Descent (basic model of MCE/GPD) are employed in the character recognition system which consists of feature extraction with filters and Euclidian distance. The learning process was applied to the similar character discrimination problem and the effects were shown as the accuracy improvement.
\end{abstract}

Key words: OCR, Feature, Filter, Learning, GPD, MCE.

\section{Introduction}

Similar character discrimination has become a primal factor to improve the OCR performance in recent years. One of the solutions for this problem is to develop an individual discrimination process for each similar pattern pair. But difficulties with this approach emerge when the number of similar pairs becomes too large. A new learning technique for fixing feature extraction parameters will be effective in such a case. This report describes a technique to fix the filter mask parameters in the feature extraction of OCR. For this purpose, Averaged Risk Criterion and Probabilistic Descent (AR/PD) were adopted as a learning method.

AR/PD was proposed by Amari [1, and improved by Katagiri and others 2 as Minimum Classification Error Criterion (MCE) with Generalized Probabilistic Descent (GPD). As the method of MCE/GPD, a simpler version which might be called AR/PD was used in this research. The learning target is the feature extraction in the system and this approach was proposed as Discriminative Feature Extraction (DFE) 3.

There are three types of target in DFE: Filter Parameter Learning, Filter Selector Learning and Filter Mask Learning. In the Filter Parameter Learning 4. [5] 6], the filters are described by some parameters like the sigma and mean value of a Gaussian filter. In the Filter Mask Learning, the learning target is the mask value of the filters. In the Filter Selector Learning [7, the filter is not used for feature extraction itself in comparison with above two approach, the filters are used for reducing the dimension of the feature vector which has been extracted by the other feature extractor.

The filter mask is the most popular technique for the feature extraction in the OCR field, and this is the reason why the Filter Mask Learning is examined in this research. 
The learning system adopted here resembles 3 layer neural nets $[8]$. The intermediate layer of those NN corresponds to the filters in the system adopted here. Also, Cognitron [9] is one of the related systems. There have been other feature learning systems [10, 11] and they have shown good performance respectively against complex and difficult problems.

Through these previous works, the filters generated in the learning system have not been considered as filters in some cases, and have not been observed visually in many cases. It has been too complex to analyze the inner workings in some cases. The purpose of this research is to investigate the learning process of feature extraction filters, the basic effect of this learning, the generated filter's performance and the human visual observation of generated filters. This report describes the successive research of the preliminary investigation 12 on this subject.

\section{Overview of a Learning System}

The recognition system used in this research is shown in Fig. 1. The local regions are assigned in the input image region and the local filter masks are defined and correspond to each local region. The inner products calculated by the local region vector consists of pixel values and local filter masks are treated as the elements of feature vector. The nonlinear transformation is executed after the inner products calculation. This feature vector is provided to the Euclidian distance unit and the distance is calculated with reference vectors.

If the local filter denoted $F$ is a linear transformation, $z$ is an input vector and $\hat{x}$ is a transformed vector, the transformation is described as $\hat{x}=F z$.

Let the element value of $x$ and $\hat{x}$ be $x_{i}$ and $\hat{x}_{i}$ respectively, the nonlinear conversion is defined as $x_{i}=\rho\left(\hat{x}_{i}\right)$ and let it be denoted as $x=\rho(\hat{x})$. By this notation, the feature extraction from $z$ is described as follows.

$$
x=\rho(F z) .
$$

If the reference vector is denoted as $\varphi$, the Euclidian distance $S$ is described as

$$
S=\|x-\varphi\|^{2} .
$$

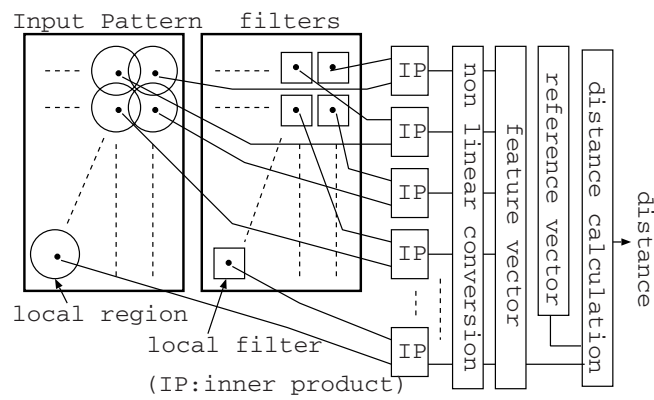

Fig. 1. Outline of recognition system used for filter mask learning 
The recognition result is determined by the category whose reference have the minimum distance.

The update terms $\Delta F, \Delta \varphi$ are calculated from $F$ and $\varphi$ by $\mathrm{AR} / \mathrm{PD}$ theory and $F$ and $\varphi$ are changed so that the system has better performance through this updating. The changed filter masks $F$ are arranged and displayed to be observed visually as shown in Fig. 1.

The update equation is obtained as described below by AR/PD. Let $l(d)$ be the loss function and $l^{\prime}(d)$ represent the differential function. Here, $d$ is defined by $d=S_{o k}-S_{e r r}$ where $S_{o k}$ is the distance between the correct category's reference and the input, and $S_{\text {err }}$ is the one between the other best category's reference and input (see (2) ). The update equations are

$$
\begin{gathered}
\Delta F=\mp 2 \epsilon_{f} l^{\prime}(d) \rho^{\prime}(\hat{x})(x-\varphi) z^{T}, \\
\Delta \varphi= \pm 2 \epsilon_{r} l^{\prime}(d)(x-\varphi),
\end{gathered}
$$

where $\epsilon_{f}, \epsilon_{r}$ are the values which define the strength of the learning. The upper part of \pm or $\mp$ is for updating when an input category is same as reference's one and the lower is for other category's input.

\section{Experiments}

System of Experiments. An input character image's size is normalized to be a 40x40 pattern and is provided to the feature extractor. Fig. 2 shows arrangements of local filter masks at the upper left portion of the input image area and the small square represents one pixel. The positions $a, \ldots, p$ means the center of each filter. The filter size is $7 \times 7$ and it is shown as a square in broken line. The filter $F$ is the filter whose center is $f$, the filter $K$ is the filter whose center is $k$. The filters are located at intervals of 3 pixels for horizontal and vertical directions. The output feature vector size is $14 \times 14$.

The initial values $f_{x, y}$ of filters are given by

$$
f_{x, y}=A \cdot \exp \left(-1 / 2\left(x^{2} / \sigma_{x}+y^{2} / \sigma_{y}\right)\right),
$$

where $(\mathrm{x}, \mathrm{y})$ is the pixel position and $A$ is a constant for normalization.

The concrete values of the initial filter used in this research are shown in Fig. 3 The upper left $4 \times 4$ pixels of the $7 \times 7$ mask which corresponds to (5) are shown. In this filter, $\sigma_{x}$ is slightly larger than $\sigma_{y}$ because it shows better performance when comparing the case of $\sigma_{x}=\sigma_{y}$ in the preliminary test. The initial reference vectors are calculated by these initial filters.

The value $\hat{x}_{i}$ is obtained as an inner product of the filter mask and the input pattern's pixel values in the local region. Then it is converted by a nonlinear function. The function adopted here is

$$
\rho\left(\hat{x}_{i}\right)=1 /\left(1+e^{\left(b-a \hat{x}_{i}\right)}\right) .
$$

The values $a, b$ are set to $a=9.4, b=2$ so that the output is from 0.12 to 0.88 when the input is from 0 to 0.42 . 


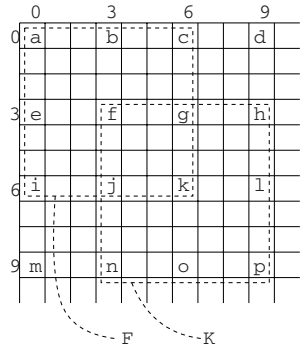

Fig. 2. Filter size and arrangement

\begin{tabular}{|l|l|l|l|l}
\hline .00000 & .00005 & .00027 & .00049 & \\
\hline .00007 & .00132 & .00769 & .01385 & \\
\hline .00051 & .00973 & .05684 & .10235 & \\
\hline .00100 & .01896 & .11070 & .19935 & \\
\hline
\end{tabular}

Fig. 3. Upper left $4 \times 4$ values of initial filter

The sigmoid function is adopted as a loss function and it's differential form are

$$
l(d)=1 /\left(1+e^{-\delta d}\right), \quad l^{\prime}(d)=\delta l(d)(1-l(d)) .
$$

Environment of Experiments. There are two similar character pairs used in the experiments, one is "bo" and "po", and the other is "ma" and "mon". These are all Japanese handwritten characters. The example of "bo" and "po" are shown in Fig. 5, 6] and the difference between those characters is only the upper right portion. The example of "ma" and "mon" are shown in Fig. 8, 9] and the difference between those characters is only the lower center portion.

There are 2000 "bo-po" patterns for learning and 2000 for testing. There are 2000 "ma-mon" patterns for learning and 1300 for testing.

The number of iteration was 3540 times for "bopo", and 2020 times for "mamon" where one cycle learning needed approximately 1.5 seconds with $2.8 \mathrm{G}$ PenIV PC.

Results of Experiment for "bo-po". Fig. 4 (A) shows the initial filters used in this research. The filters are arranged with 14 pieces horizontally and 14 pieces vertically (just 4 columns are shown). Each of the filters has $7 \times 7$ pixels. The pixel values are represented by a black circle. A large black circle means a large plus value and a small black circle means a large minus value. The value zero corresponds to the circles observed at the rim of the filters. Here, even if the filters in the figure seem to be slightly different, all filters are the same and this might be the effect of image capture and printing.

Fig. 4 (B) shows the generated filters after learning. Almost all filters except the filters located in the upper right corner seem to be the same as the initial filter. The generated filters in the upper right corner are considered to be the vertical line extraction filter. They resemble a Gabor filter or a Mexican hat filter. These results show that the learning has made the filter emphasize the difference between the two category images.

Fig. 5 and 6 show the input patterns in the upper array of the figure, the feature patterns obtained by initial filters in the middle array, and the feature patterns obtained by generated filters in the bottom array. The feature patterns 


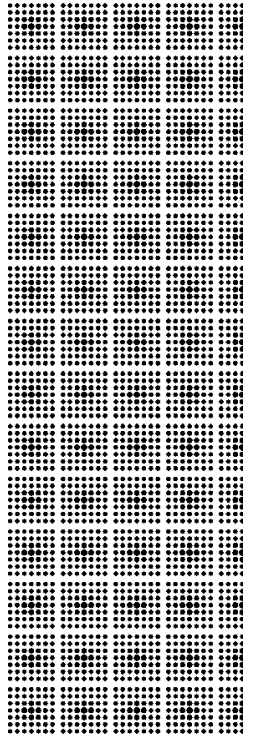

(A)

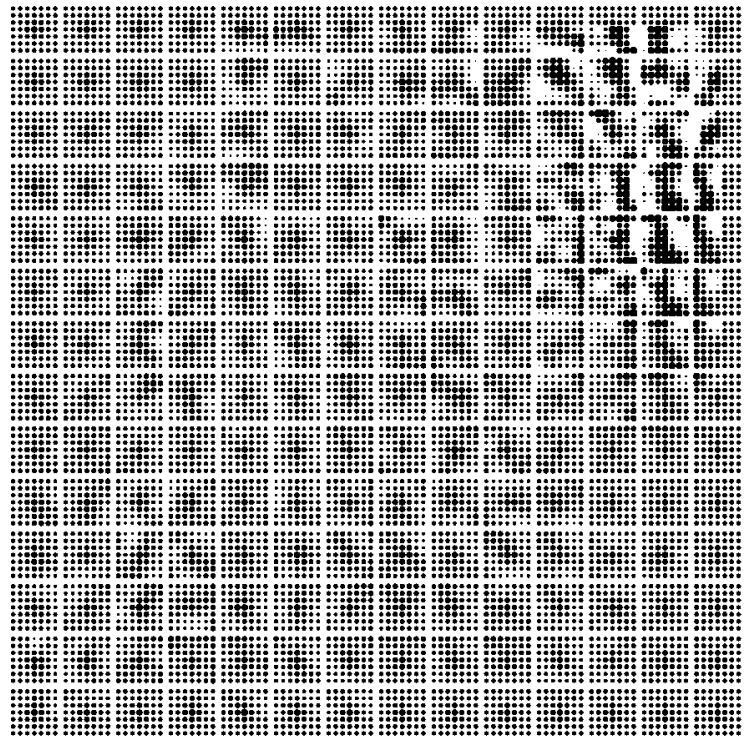

(B)

Fig. 4. (A) Initial filters before learning (left side part: 4 columns), (B) Filters generated by "bo", "po" character learning

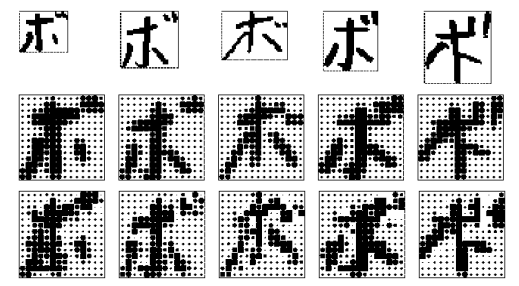

Fig. 5. "bo": Original (upper), Gauss (middle), Generated (bottom)

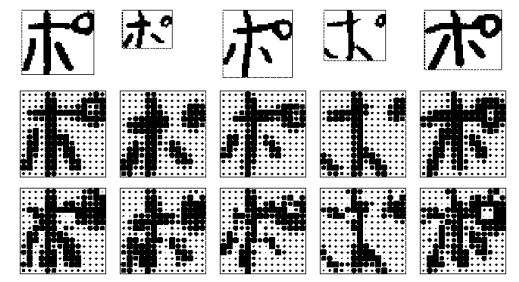

Fig. 6. "po": same order as the patterns on the left ("bo")

become noisy and the right upper part is emphasized more after learning. Some of the "bo" pattern's two dots in the right upper part are separated while they are touching in the initial one. In the "po" case, the upper part seems slightly larger than the initial one.

Results of Experiment for "ma-mon". Fig. 7(B) shows the generated filters after learning. Fig. 8 and 9 shows the input patterns and feature patterns in the same manner as the "bo-po" results. In these figures, the lower center part of the generated patterns seem to be emphasized and they are noisy compared with the initial patterns. 


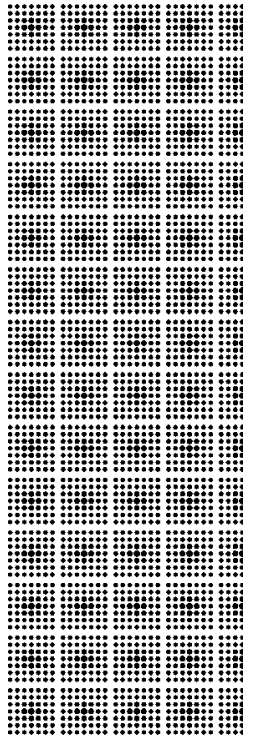

(A)

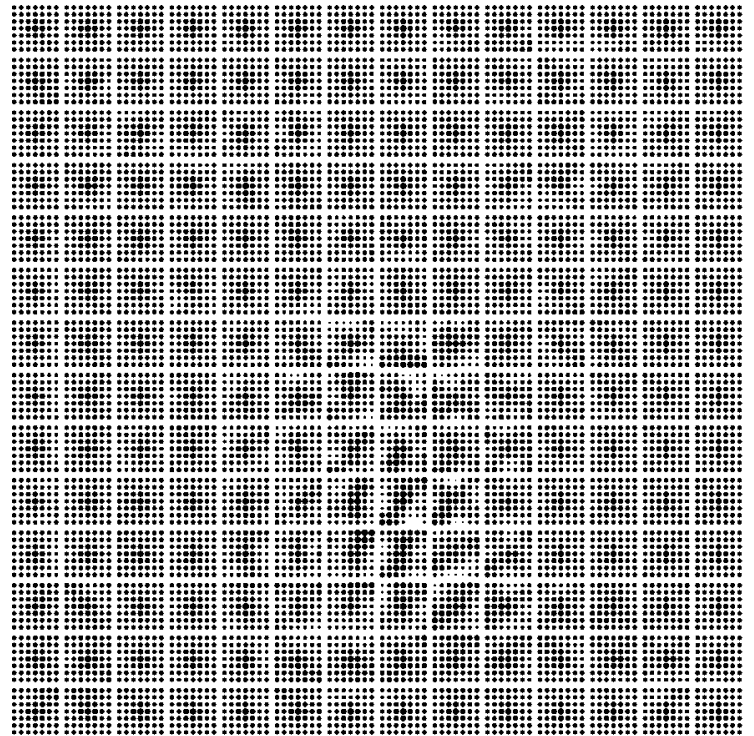

(B)

Fig. 7. (A) Initial filters before learning (left side part: 4 columns), (B) Filters generated by "ma", "mon" character learning

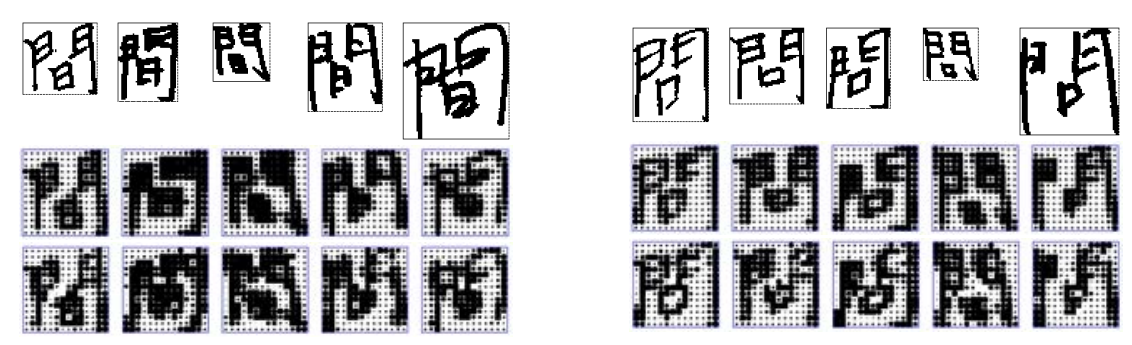

Fig. 8. "ma": Original (upper), Gauss

Fig. 9. "mon":same order as the pat(middle), Generated (bottom) terns on the left ("ma")

Evaluation of Performance of Generated Filters. In the case of "bo-po" discrimination, the learning performance in terms of recognition accuracy was tested by comparing it to the Gabor feature extraction [13. The Gabor feature extraction adopted here has a simple structure that has $8 \times 8$ local regions and 4 directions in each local region, totaling 256 output elements. This kind of Gabor filter is often used for character recognition and considered to have reasonable processing speed and good performance. As for the recognition method, LVQ, SVM, MQDF (modified quadratic discriminant functions) were tested. LVQ 14 ] is the version of a differentiated sigmoid window function, the system "svmlight" [15] is used as SVM and MQDF is constructed based on the reference [16. 
Table 1. Error rate for "bo-po"

\begin{tabular}{|c|c|c|c|}
\hline Method & Gauss & Learning & Gabor \\
\hline \hline FML & & $2.7 \%$ & \\
\hline \hline LVQ & $6.2 \%$ & $2.8 \%$ & $3.8 \%$ \\
\hline SVM & $5.5 \%$ & $2.7 \%$ & $3.9 \%$ \\
\hline MQDF & $6.6 \%$ & $3.2 \%$ & $4.7 \%$ \\
\hline \hline Average & $6.1 \%$ & $2.9 \%$ & $4.1 \%$ \\
\hline
\end{tabular}

Table 2. Error rate for "ma-mon"

\begin{tabular}{|c|c|c|c|}
\hline Method & Gauss & Learning & Gabor \\
\hline \hline FML & & $8.3 \%$ & \\
\hline \hline LVQ & $12.8 \%$ & $8.2 \%$ & $16.1 \%$ \\
\hline SVM & $8.7 \%$ & $7.3 \%$ & $11.5 \%$ \\
\hline MQDF & $12.9 \%$ & $9.8 \%$ & $14.3 \%$ \\
\hline \hline Average & $11.5 \%$ & $8.4 \%$ & $14.0 \%$ \\
\hline
\end{tabular}

The results of "bo-po" are shown in Table. 1. The values in the table are the error rate. The column labeled "Gauss" means the filter is Gaussian, that is the initial filter. "Learning" means the filter is the filter obtained by filter mask learning. "Gabor" means the filter is Gabor. The array of "FML" shows the results of the filter mask learning, consequently it shows the system's performance of itself after learning.

The array of "LVQ" shows the results of LVQ with 3 types of filters, and "SVM", "MQDF" are the same. The final "Average" shows the average results of LVQ, SVM and MQDF.

From this comparison, "FML" had better performance compared to the conventional recognition system with a Gabor filter. And also, as for the filter itself, the "FML" filter had better performance compared with the Gabor filter in 3 different recognition systems.

These results are considered reasonable because the portion where the similar patterns are different is strongly focused in the generated filters. The results for "ma-mon" are also shown in Table. 2 .

\section{Parameter Setting}

Relation Between $\epsilon_{f}$ and $\epsilon_{r}$. The parameter setting is important to make a learning process stable and effective, especially the parameters $\epsilon_{f}$ and $\epsilon_{r}$ are essential. Both parameters have a relationship to each other and need to have different values in some cases for effective learning.

Fig. 10 shows a simple evaluation function as an example. This figure represents a contour map of some function on the $x-y$ plane. The lower value is better and position $\mathrm{B}$ has the lowest value. Let the start position be $A$, and let the optimization process be a descent algorithm. In this case, the first direction to change position is shown by arrow $C$ which is the normal line to the contour line. In this figure, let's imagine that the ellipse's long axis is 1000 times longer than the short axis, like the Grand Canyon. It might be difficult to come close to $B$ from $A$ because there is too much distance between $A$ and $B$ in comparison with the adequate length of $C$ for stable learning. The direction of $C$ is completely different from the desired direction. This problem could be related to the research [17, but a simple approach is adopted here. 


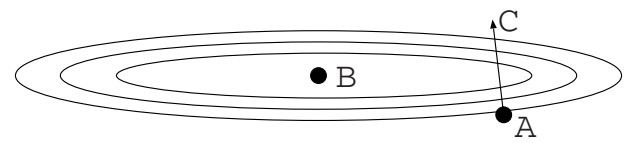

Fig. 10. Grand Canyon problem in descent algorithm

To avoid this problem, we need to convert such a super thin ellipse to the adequate size ellipse to avoid this difficulty. This conversion is virtually realized by the technique in which the parameters $\epsilon_{f}$ and $\epsilon_{r}$ have different values.

For this purpose, the proper value of $\epsilon$ was estimated as follows. The elements of the update filter (3) and vector (4) are described as

$$
\Delta F_{i j}=\mp 2 \epsilon_{f} l^{\prime}(d) \rho^{\prime}\left(\hat{x_{i}}\right)\left(x_{i}-\varphi_{i}\right) z_{j}, \quad \Delta \varphi_{i}= \pm 2 \epsilon_{r} l^{\prime}(d)\left(x_{i}-\varphi_{i}\right) .
$$

Here, $\overline{|a|}$ denotes the estimated average of absolute value of $a$, and $\left\langle\left\langle F_{i j}\right\rangle\right\rangle$, $\left\langle\left\langle\Delta F_{i j}\right\rangle\right\rangle,\left\langle\left\langle\varphi_{i}\right\rangle\right\rangle$ and $\left\langle\left\langle\Delta \varphi_{i}\right\rangle\right\rangle$ are defined by follows. $\left\langle\left\langle F_{i j}\right\rangle\right\rangle=\overline{\left|F_{i j}\right|},\left\langle\left\langle\varphi_{i}\right\rangle\right\rangle=\overline{\left|\varphi_{i}\right|}$,

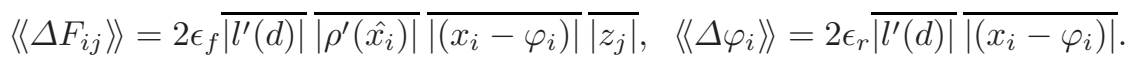

These estimated average values are determined by human observation of experimental data. The following results are obtained by those values.

$$
\left\langle\left\langle F_{i j}\right\rangle\right\rangle=0.2, \quad\left\langle\left\langle\Delta F_{i j}\right\rangle\right\rangle=1200 \epsilon_{f}, \quad\left\langle\left\langle\varphi_{i}\right\rangle\right\rangle=32, \quad\left\langle\left\langle\Delta \varphi_{i}\right\rangle\right\rangle=8 \epsilon_{r} .
$$

The final result $\epsilon_{f} / \epsilon_{r}$ is calculated by (10) and the assumption (11).

$$
\begin{gathered}
\left\langle\left\langle\Delta F_{i j}\right\rangle\right\rangle /\left\langle\left\langle F_{i j}\right\rangle\right\rangle=\left\langle\left\langle\Delta \varphi_{i}\right\rangle\right\rangle /\left\langle\left\langle\varphi_{i}\right\rangle\right\rangle . \\
\epsilon_{f} / \epsilon_{r}=40 \times 10^{-6} .
\end{gathered}
$$

This estimated result is compared with the experimental results in Fig. 11 Several values of $\epsilon_{r}$ are tested with a certain value of $\epsilon_{f}$, and the best value of $\epsilon_{r}$ is determined for each value of $\epsilon_{f}$. This best value is calculated as the average of the good values with which the system has showed good performance. These best values are plotted in Fig. 11 as "result". The line "estimated" refers to equation (12). This estimation fits well to the obtained values as the best values up to the value $\epsilon_{r}=0.03$.

As a result, the above mentioned approach was proved to be useful to estimate the ratio of $\epsilon$.

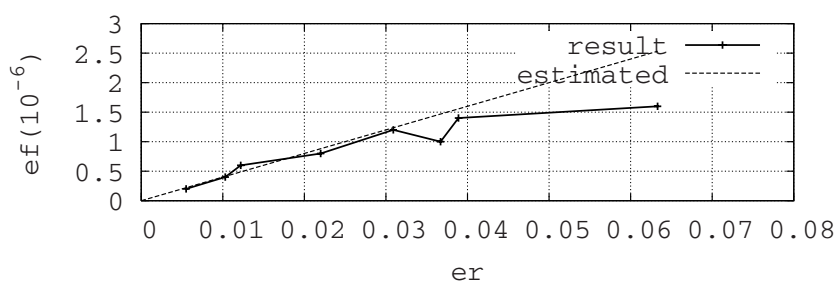

Fig. 11. Estimated $\epsilon_{f} / \epsilon_{r}$ and the experimental results 
Fixing $\delta$ in Sigmoid Function. The recognition accuracy after learning depends on the parameter $\delta$ in (7). This value is determined by human observation on the distribution of value $d$ obtained from the actual recognition system. This value was determined so that about $1 / 5$ or $1 / 10$ of the patterns contribute to the learning. After that, several values around the above determined value are tested in preliminary attempts, and the best value is finally determined as $\delta$.

\section{Conclusion}

Through these experiments, the AR/PD approach for Filter Mask Learning was proved to be effective in terms of the system's recognition accuracy and the generated filters had good performance even if it was embedded in the other recognition systems. The filter images and the feature patterns prepared for human observation showed that the learning was focused on the important part for discrimination of similar patterns.

The multiple filters at the local regions and the multi layer filter structure will be considered in the further research and they are expected to become useful technology in pattern recognition and provide new knowledge about intelligence and learning.

\section{References}

1. Amari, S.: A Theory of Adaptive Pattern Classifiers. IEEE Trans. EC 16(3), 299307 (1967)

2. Katagiri, S., Lee, C.-H., Juang, B.-H.: A Generalized Probabilistic Descent Method, ASJ, Fall Conf. 2-p-6, Nagoya, Japan pp. 141-142 (1990)

3. Paliwal, K.K., Bacchiani, M., Sagisaka, Y.: Minimum Classification Error Training Algorithm for Feature Extractor and Pattern Classifier in Speech Recognition. EUROSPEECH '95 1, 541-544 (1995)

4. Biem, A., Katagiri, S.: Feature Extraction Based on Minimum Classification Error / Generalized Probabilistic Descent Method. In: Proc. IEEE Int. Conf. Acoust. Speech, Signal Processing 2, 275-278 (1993)

5. Biem, A., Katagiri, S.: Filter Bank Design Based on Discriminative Feature Extraction. In: Proc. IEEE Int. Conf. Acoust. Speech, Signal Processing 1, 485-488 (1994)

6. Bacchiani, M., Aikawa, K.: Optimization of Time-Frequency Masking Filters Using the Minimum Classification Error Criterion. In: Proc. IEEE Int. Conf. Acoust. Speech, Signal Processing 2, 197-200 (1994)

7. Kawamura, A. and Nitta, T.: Feature-Extraction-Based Character Recognition Using Minimum Classification Error Training, IEICE Trans. Information and Systems, Vol.J81-D-II, No.12 2749-2756, (in Japanese) (1998)

8. LeCun, Y., Bottou, L., Haffner, P.: Gradient-Based Learning Applied to Document Recognition. In: Proc. of the IEEE 86(11), 2278-2324 (1998)

9. Fukushima, K.: Neocognitron: a Self-Organizing Neural Network Model for a Mechanism of Pattern Recognition Unaffected by Shift in Position. Biol. Cybernet. 36, 193-202 (1980) 
10. Lampinen, J., Oja, E.: Distortion Tolerant Pattern Recognition Based on SelfOrganizing Feature Extraction. IEEE Trans. Neural Networks 6(3), 539-547 (1995)

11. Koichi, I., Tanaka, H., Tanaka, K., Kyuma, K.: Learning Algorithm by Reinforcement Signals for the Automatic Recognition System, SMC2004 pp. 4844-4848 (2004)

12. Kurosawa, Y.: Filter Learning Method for Feature Extraction in Character Recognition System, MIRU2006, (in Japanese) (2006)

13. Hamamoto, Y., Uchimura, S., Watanabe, M., Yasuda, T., Mitani, Y., Tomita, S.: A Gabor Filter-Based Method for Recognizing Handwritten Numerals, Pattern Recognition 31(4), 395-400 (1998)

14. Kohonen, T.: Learning Vector Quantization, Helsinki University of Technology, Laboratory of Computer and Information Science, Report TKK-F-A601 (1986)

15. Joachims, T.: Making Large-Scale SVM Learning Practical, Advances in Kernel Methods - Support Vector Learning. MIT-Press, Cambridge (1999)

16. Kimura, F., Takashina, K., Tsuruoka, S., Miyake, Y.: Modified Quadratic Discriminant Functions and the Application to Chinese Character Recognition. IEEE Trans. PAMI 9(1), 149-153 (1987)

17. Amari, S.: Natural Gradient Works Efficiently in Learning, Neural Computation, Vol. Neural Computation 10(2), 251-276 (1998) 